\title{
HIGHER $K$-THEORY FOR REGULAR SCHEMES
}

\author{
BY S. M. GERSTEN
}

Communicated by Morton L. Curtis, July 17, 1972

\begin{abstract}
Higher $K$-groups are defined for regular schemes, generalizing the $K$-theory of Karoubi and Villamayor. A spectral sequence is developed which shows how the $K$-groups depend on the local rings of the scheme. Applications to curves and affine surfaces are given.
\end{abstract}

Let $X$ be a regular separated scheme. If $U$ is an affine open subset of $X$, then the assignment $U \mapsto \operatorname{BGl}\left(S^{n} \Gamma\left(U, O_{X}\right)_{*}\right)$ is a sheaf of Kan complexes on the Zariski site. Here $S$ denotes the suspension ring functor of Karoubi [10] and if $A$ is a ring, $A_{*}$ denotes the simplicial ring [11]

$$
\left(A_{*}\right)_{n}=A\left[t_{0}, t_{1}, \ldots, t_{n}\right] /\left(t_{0}+\cdots+t_{n}-1\right) .
$$

We recall that $\pi_{i} \mathrm{BGl} A_{*}=K^{-i} A, i \geqq 1[11]$, where the $K$-groups of Karoubi and Villamayor are indicated [10]. Also, recall that $K_{0}(A)$ $\times \operatorname{BGl}\left(A_{*}\right) \simeq \operatorname{\Omega BGl}\left(S A_{*}\right)$ if $A$ is $K$-regular ([9], [8]). Thus there is a sheaf of Kan spectra $E\left(O_{X}\right)$ on $X$ associated to the pre-spectrum $U \mapsto$ $\left(n \mapsto \operatorname{BGl}\left(S^{n} \Gamma\left(U, O_{X}\right)_{*}\right)\right)$. Such sheaves have been studied by $\mathrm{K}$. Brown [4] who has defined cohomology with coefficients in a sheaf of Kan spectra: $H^{n}\left(X, E\left(O_{X}\right)\right), n \in Z$.

Definition. $K^{n}(X)=H^{n}\left(X, E\left(O_{X}\right)\right)$.

We remark that the spectra $E\left(O_{X}\right)$ are connected since $X$ is regular, so $K^{i}(X)=0$ if $i>0$. The main properties of these groups and most of the motivation for introducing them are summarized in

THEOREM 1. Let $X$ be a regular separated scheme.

(1) If $U$ and $V$ are open subschemes of $X$, then there is an exact MayerVietoris sequence

$$
\cdots \rightarrow K^{i-1}(U \cap V) \rightarrow K^{i}(U \cup V) \rightarrow K^{i}(U) \oplus K^{i}(V) \rightarrow K^{i}(U \cap V) \rightarrow \cdots
$$

(2) If $X$ has finite (Krull) dimension, then there is a fourth quadrant spectral sequence of cohomological type

$$
E_{2}^{p q}=H^{p}\left(X, \underline{K}^{q}\right) \Rightarrow K^{p+q}(X) .
$$

Here $K^{q}$ is the sheaf in the Zariski site associated to the presheaf

$$
U \mapsto K^{q}\left(\Gamma\left(U, O_{X}\right)\right), \quad U \text { affine open. }
$$

AMS (MOS) subject classifications (1970). Primary 18F25, 55B15, 16A54, 13D15, $55 \mathrm{~F} 50,18 \mathrm{G} 30,55 \mathrm{~B} 20,55 \mathrm{D} 35$. 
(3) If $X=\operatorname{spec} A$, then $K^{i}(X)=K^{i}(A)$, the Karoubi-Villamayor $K$-groups of $A$.

(4) $K^{i}\left(X \times{ }_{\operatorname{spec} Z} \operatorname{spec} Z[t]\right)=K^{i}(X)$.

The properties (1) and (2) are formal properties of sheaves of Kan spectra [4]. Property (4) is immediate, since the Karoubi-Villamayor theory is invariant under polynomial extension. Property (3) however is a theorem whose proof depends on the main results of [7]. In addition the Krull theory of divisors enters in the description of the affine opens of spec $A$. Full details will be published elsewhere.

Properties (1) and (3) actually serve to provide an axiomatic characterization for the theory, in the category of regular separated noetherian schemes, as a simple induction argument shows. Also, since $K^{n}(X)$ arise as the homotopy of a spectrum, these groups are the coefficient groups (cohomology of a point) in a generalized cohomology theory of complexes.

COROLLARY 2. If $X$ is a regular curve, then there are short exact sequences

$$
0 \rightarrow H^{1}\left(X, \underline{K}^{-n-1}\right) \rightarrow K^{-n}(X) \rightarrow H^{0}\left(X, \underline{K}^{-n}\right) \rightarrow 0, \text { all } n \geqq 0 .
$$

This is merely the fact that the spectral sequence of Theorem 1 degenerates at the $E_{2}$ level for curves.

Proposition 3. If $A$ is a Dedekind ring with field of fractions $F$, then the sequence

$$
K_{2}(A) \rightarrow K_{2}(F) \rightarrow \coprod_{\underline{m} \in \max A} K_{1}(A / \underline{m}) \rightarrow K_{1}(A) \rightarrow \cdots
$$

is exact.

REMARK. Exactness at $K_{2} F$ was shown by Bass if $A$ has only countably many maximal ideals [2]. Exactness at other points is classical.

One makes use of the recently discovered short exact sequence of K. Dennis and M. R. Stein [6] to construct a short exact sequence of sheaves

$$
0 \rightarrow \underline{K^{-2}} \rightarrow \underline{K_{2} F_{X}} \rightarrow \coprod_{v} K_{1}\left(k_{v}\right) \rightarrow 0 .
$$

Here $K_{2} F_{X}$ is the constant sheaf where $F$ is the field of rational functions of $X$, and $\coprod_{v} K_{1}\left(k_{v}\right)$ assigns to each open set $U$ the group

$$
\coprod_{v \in U ; v \text { closed }} K_{1}\left(k_{v}\right)
$$

where $k_{v}$ is the residue class field at $v$. One takes the long exact cohomology sequence associated to this short exact sequence of sheaves, and splices it to the short exact sequences of Corollary 2 to get the result. 
Proposition 4. Let $X$ be a regular affine surface and suppose that $\xi$ is a vector bundle on $X$. Suppose in addition that det $\xi$, the determinant bundle in Pic $X$, is trivial. Then $\xi$ and rank $\xi$ have the same class in $K^{0}(X)$ if and only if $c_{2}(\xi)=0$, where $c_{2}(\xi) \in H^{2}\left(X, \underline{K}^{-2}\right)$ is the universal second Chern class of $\xi$.

The interpretation of the class of $\xi$ in $H^{2}\left(X, \underline{K}^{-2}\right)$ as a universal Chern class is suggested by recent work of Spencer Bloch. This result follows from the spectral sequence of Theorem 1 with the observation that the differential $d_{2}: H^{0}\left(X, \underline{K}^{-1}\right) \rightarrow H^{2}\left(X, \underline{K}^{-2}\right)$ is zero, since $H^{0}\left(X, \underline{K}^{-1}\right)$ $=\Gamma\left(X, O_{X}^{*}\right)=U(A)$ is a direct factor of $K^{-1}(X)=K_{1}(A)$, where $A=$ $\Gamma\left(X, O_{X}\right)$

Denote now by $K_{i}^{\mathrm{ab}}(X), i=0,1$, the $K$-groups of the abelian category of coherent $O_{X}$ modules [1]. There are natural morphisms $K_{i}^{\mathrm{ab}} \rightarrow K^{-i}$. Of course, by Theorem $1, K_{i}^{\mathrm{ab}}(X)=K^{-i}(X)$ if $X$ is affine and regular $(i=0,1)$. From the Mayer-Vietoris sequence of Theorem 1 and the corresponding Mayer-Vietoris sequence for $K_{0}^{\mathrm{ab}}$ (which can be deduced from [5, Proposition 7]), it follows that if the regular scheme $X$ is the union of two open affines, then $K_{0}^{\mathrm{ab}}(X)=K^{0}(X)$. In particular this holds for curves. However, we do not know how much more generally this result holds.

Concerning $K_{1}^{\mathrm{ab}}$ the result is less satisfactory. Using the results of $\mathrm{L}$. Robert's thesis [12] we can show

PROPOSITION 5. If $X$ is a complete nonsingular elliptic curve over the complex numbers, then $K_{1}^{\mathrm{ab}}(X) \rightarrow K^{-1}(X)$ is surjective but not injective.

If $X$ is a complete nonsingular curve over the constant field $k$, the algebraic closure of a finite field, then using results of Tate [13] we can show that $K^{-2}(X)=\operatorname{Tor}\left(k^{*}, \operatorname{Pic}(X)\right)$ and $K^{-1}(X)=k^{*} \otimes \operatorname{Pic} X \cong k^{*}$. The first assertion amounts to an identification of $K^{-2}(X)$ with the tame kernel in the function field case.

\section{REFERENCES}

1. H. Bass, Algebraic K-theory, Benjamin, New York, 1968. MR 40 \# 2736.

2. $-K_{2}$ des corps globaux, Séminaire Bourbaki 1971, Exposé 394.

3. K. Brown, Thesis, M.I.T., Cambridge, Mass., 1971.

4. - Abstract homotopy theory and generalized sheaf cohomology (preprint).

5. A. Borel and J. P. Serre, Le théorème de Riemann-Roch, Bull. Soc. Math. France 86 (1958), 97-136. MR 22 \#6817.

6. K. Dennis and M. R. Stein, A new exact sequence for $K_{2}$ and some consequences for rings of integers, Bull. Amer. Math. Soc. 78 (1972), 600-603.

7. S. M. Gersten, A Mayer-Vietoris sequence in the K-theory of localizations. J. Pure Appl. Algebra (to appear).

8. - On the spectrum of algebraic K-theory, Bull. Amer. Math. Soc. 78 (1972), 216-219.

9. - The relationship between the K-theory of Karoubi and Villamayor and the Ktheory of Quillen (preprint). 
10. M. Karoubi and O. Villamayor, Foncteurs $K^{n}$ en algèbre et en topologie, C. R. Acad. Sci. Paris Sér. A-B 269 (1969), A416-A419. MR 40 \# 4944.

11. D. L. Rector, The K-theory of a space with coefficients in a discrete ring, Bull. Amer. Math. Soc. 77 (1971), 571-575.

12. L. Roberts, Thesis, Harvard University, Cambridge, Mass., 1968.

13. J. Tate, Symbols in arithmetic, Proc. Internat. Congress Math. (Nice, 1970), vol. 1, Gauthier-Villars, Paris, 1971, pp. 201-211.

Department of Mathematics, Rice University, Houston, Texas 77001 